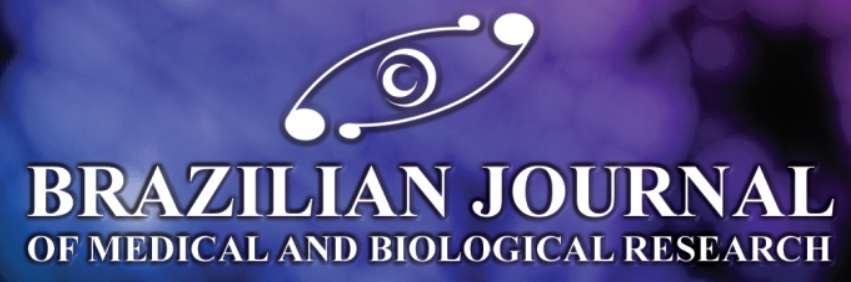
www.bjournal.com.br
ISSN 1414-431X

Volume 45 (11) 995-1101 November 2012

\section{BIOMEDICAL SCIENCES}

AND

CLINICAL INVESTIGATION

Braz J Med Biol Res, November 2012, Volume 45(11) 1074-1079

doi: 10.1590/S0100-879X2012007500135

Evaluation of chromosomal abnormalities by cIg-FISH and association with proliferative and apoptotic indexes in multiple myeloma

C.C.G. Linardi, G. Martinez, E.D.R.P. Velloso, A.M. Leal, C.A. Kumeda, V. Buccheri, R.S. Azevedo, L.M. Peliçario and P. Dorlhiac-Llacer

The Brazilian Journal of Medical and Biological Research is partially financed by

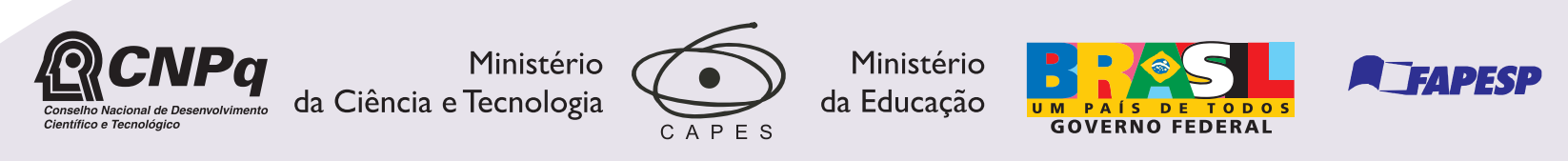
Institutional Sponsors

sing 0
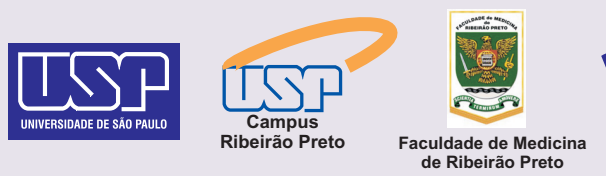

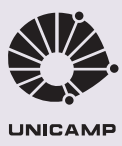

SHIMADZU

Associaçăa
Fundo de Incentivo
à Pesquisa

xplore High - Performance MS Orbitrap Technology $\underset{\text { analitice Thermo }}{\text { and }}$ 


\title{
Evaluation of chromosomal abnormalities by clg-FISH and association with proliferative and apoptotic indexes in multiple myeloma
}

\author{
C.C.G. Linardi ${ }^{1}$, G. Martinez¹, E.D.R.P. Velloso ${ }^{1}$, A.M. Leal ${ }^{1}$, C.A. Kumeda1, \\ V. Buccheri ${ }^{1}$, R.S. Azevedo ${ }^{2}$, L.M. Peliçario ${ }^{1}$ and P. Dorlhiac-Llacer ${ }^{1}$ \\ ${ }^{1}$ Disciplina de Hematologia e Hemoterapia, Departamento de Clínica Médica, \\ Hospital das Clínicas, Faculdade de Medicina, Universidade de São Paulo, São Paulo, SP, Brasil \\ 2Departamento de Patologia, Hospital das Clínicas, Faculdade de Medicina, \\ Universidade de São Paulo, São Paulo, SP, Brasil
}

\begin{abstract}
Eighty-six newly diagnosed multiple myeloma (MM) patients from a public hospital of São Paulo (Brazil) were evaluated by clgFISH for the presence of del(13)(q14), $\mathrm{t}(4 ; 14)(\mathrm{p} 16.3 ; \mathrm{q} 32)$ and del(17)(p13). These abnormalities were observed in 46.5, 9.3, and $7.0 \%$ of the patients, respectively. In order to identify the possible role of del(13)(q14) in the physiopathology of MM, we investigated the association between this abnormality and the proliferative and apoptotic indexes of plasma cells. When cases demonstrating $\mathrm{t}(4 ; 14)(\mathrm{p} 16.3 ; q 32)$ and $\operatorname{del}(17)(\mathrm{p} 13)$ were excluded from the analysis, we observed a trend towards a positive correlation between the proportion of cells carrying del(13)(q14) and plasma cell proliferation, determined by Ki-67 expression $(r=0.23, \mathrm{P}=0.06)$. On the other hand, no correlation between the proportion of cells carrying del(13)(q14) and apoptosis, determined by annexin- $V$ staining, was detected $(r=0.05, P=0.69)$. In general, patients carrying del(13)(q14) did not have lower survival than patients without del(13)(q14) $(P=0.15)$, but patients with more than $80 \%$ of cells carrying del(13)(q14) showed a lower overall survival $(P=0.033)$. These results suggest that, when del(13)(q14) is observed in a high proportion of malignant cells, it may have a role in determining MM prognosis. Another finding was a statistically significant lower overall survival of patients with $t(4 ; 14)(p 16.3 ; q 32)(P=0.026)$. In the present study, almost half the patients with $t(4 ; 14)(p 16.3 ; q 32)$ died just after diagnosis, before starting treatment. This fact suggests that, in São Paulo, there may be even more patients with this chromosomal abnormality, but they probably die before being diagnosed due to unfavorable socioeconomic conditions. This could explain the low prevalence of this chromosomal abnormality observed in the present study.
\end{abstract}

Key words: Multiple myeloma; Cell proliferation; Apoptosis; Prognosis; Fluorescence in situ hybridization

\section{Introduction}

Despite recent progress in the management of multiple myeloma (MM), this neoplasm remains incurable and is characterized by a heterogeneous prognosis. The variable prognosis of the disease involves interaction between intrinsic features of the disease biology such as chromosomal abnormalities and host factors. Del(13)(q14) is detected in almost $50 \%$ of cases and is associated with lower survival only when other high-risk genetic features such as del(17)(p13) and $t(4 ; 14)(p 16.3 ; q 32)$ are present $(1,2)$. However, this does not mean that del(13) (q14) has no biological importance since data suggest that this abnormality is a prerequisite for clonal expansion $(1,2)$.

The aim of the present study was to determine the prevalence of the most relevant chromosomal abnormalities and their impact on prognosis in a group of MM patients from a public hospital in São Paulo. Although these abnormalities have been studied extensively, there are few studies focusing on their prevalence in developing countries (3-5). We also investigated the association between $\operatorname{del}(13)(q 14)$ and the proliferative and apoptotic indexes of bone marrow plasma cells (PC) in order to study the possible role of this abnormality in the biology of the disease.

\section{Patients and Methods}

\section{Patients}

Bone marrow aspirates from 92 recently diagnosed

Correspondence: C.C.G. Linardi, Disciplina de Hematologia e Hemoterapia, Departamento de Clínica Médica, Hospital das Clínicas, FM, USP, Av. Dr. Eneas C. Aguiar, 155, 1ำ andar, Laboratório de Biologia Tumoral, 05403-000 São Paulo, SP, Brasil.

Fax: +55-11-3061-5544. E-mail: camilalinardi@gmail.com

Received February 20, 2012. Accepted August 1, 2012. Available online August 24, 2012. Published October 5, 2012. 
MM patients were obtained with a heparin-coated syringe. All patients were treated at Divisão de Hematologia, Hospital das Clínicas, Universidade de São Paulo, and were diagnosed consecutively between February 2007 and April 2011. The Institutional Ethics Review Board approved the study (protocol No. 016/06) and written informed consent was obtained from all patients allowing the specimen to be used for research purposes.

Patients received conventional dose induction chemotherapy consisting of a melphalan- or dexamethasonebased \pm thalidomide regimen (6). Fourteen patients received high-dose chemotherapy followed by autologous peripheral blood stem-cell support (ASCT) (6). The median follow-up was 37.3 months (0-54.8 months).

\section{Fluorescence in situ hybridization (FISH) studies}

Cytospin slides were prepared after enrichment of mononuclear cells, fixed in $95 \%$ ethanol for $5 \mathrm{~min}$ at room temperature and stored at $-20^{\circ} \mathrm{C}$ for future use. We used clg-FISH with light chain-specific immunofluorescent detection of clonal PC (7). Del(13)(q14) was determined with a retinoblastoma gene-1 LSI RB1 probe (Vysis, USA), $\operatorname{del}(17)(\mathrm{p} 13)$ with an LSI p53 probe (Vysis) combined with a chromosome 17 centromeric probe (Vysis), and $t(4 ; 14)$ (p16.3;q32) with an LSI IGH/FGFR3 probe (Vysis). Two independent observers read blindly and independently each slide, counting a total of 200 nuclei per sample. The cut-off values (fusion probe $=10 \%$, numerical abnormalities $=20 \%$ ) adopted were in accordance with the recommendations of the European Myeloma Network FISH workshop (8).

\section{Flow cytometry}

Monoclonal antibodies (MoAb) anti-CD138-PE (DAKO, Denmark), anti-CD38-APC (eBioscience, USA) and antiCD45-PC5 (Immunotech-Beckman Coulter, France) were used to identify bone marrow PC. To detect proliferative cells, membrane cells were permeabilized with saponin and cells were stained with the anti-Ki-67 MoAb (Ki-67 FITC, Pharmingen, USA) (9). The Annexin-V-FITC kit (DAKO, The Netherlands) was used to detect phosphatidylserine expression on the surface of apoptotic cells after enrichment of mononuclear cells using the Ficoll-gradient centrifugation method. All acquisitions were performed using a FACSCalibur flow cytometer (BD Biosciences, USA) and the CellQuest program (BD Biosciences) and at least $2 \mathrm{x}$ $10^{3} \mathrm{PC} /$ tube were recorded.

\section{Statistical analysis}

Differences among groups were determined using the Fisher exact test for categorical variables. Differences among groups were determined using the Kruskal-Wallis test for continuous variables. Correlations between continuous variables were investigated using the nonparametric Spearman test.

Overall survival (OS) was calculated from the time of diagnosis to death from any cause or last contact using the Kaplan-Meier method. Event-free survival (EFS) was calculated from the time of diagnosis to progression, loss of response or death from any cause, or last contact, using the Kaplan-Meier method. The survival curves were compared by the log-rank test. All $P$ values were 2-tailed and the alpha error was defined as $5 \%$. The statistical analyses were performed using the Stata Statistical Software, Release 11 (StataCorp LP, USA).

\section{Results}

\section{Characteristics of patients at diagnosis}

The median age of the 92 patients was 63.5 years (range $=36-93$ ); $51.2 \%$ were males .

Sixty-four percent of patients had an IgG paraprotein, $20.2 \%$ had an $\operatorname{lgA}$ paraprotein, $12.3 \%$ had light chains only (kappa $=5.6 \%$ and lambda $=6.7 \%$ ), and $3.4 \%$ were non-secretory. Fifty percent of patients were classified as International Staging System (ISS) (10) stage III, 36.6\% as stage II, and $13.4 \%$ as stage I. The clinical and laboratory features of the patients are summarized in Table 1.

\section{Prevalence of chromosomal abnormalities}

The chromosomal abnormalities $\mathrm{t}(4 ; 14)(\mathrm{p} 16.3 ; \mathrm{q} 32)$, $\operatorname{del}(17)(p 13)$ and del(13)(q14) were studied in 86 patients and were detected in $8(9.3 \%), 6(7 \%)$, and $40(46.5 \%)$ of them, respectively. There were no significant differences in age or gender between the different groups. The median age of patients with $\mathrm{t}(4 ; 14)(\mathrm{p} 16.3 ; \mathrm{q} 32)$, del(17)(p13), and with only del(13)(q14) was 59 (range = 54-73), 57.5 (range $=43-76)$, and 65 (range $=36-79)$, respectively. Forty patients with a median age of 63.5 years (range $=$ 36-93) did not have any of the abnormalities evaluated in this study. In 6 of 92 patients, it was not possible to study the different chromosomal abnormalities due to hybridization failure.

In del(13)(q14)-positive cases, the median number of PC with this abnormality was $75 \%$ (range $=28-98 \%$ ). In $\mathrm{t}(4 ; 14)$ (p16.3; $\mathbf{3 2})$-positive cases, the median number of PC with this abnormality was $84 \%$ (range $=76-94 \%$ ), and 6 of these patients also had del(13)(q14). In del(17) (p13)-positive cases, the median number of PC with this abnormality was $35 \%$ (range $=21-76 \%$ ) and 2 of these patients also had del(13)(q14).

The majority of patients $(84.8 \%)$ were treated just with conventional doses of chemotherapy. Only 14 patients received ASCT: 8 of them with at least one of the abnormalities studied, and 6 of them without any of these chromosomal alterations. The characteristics of patients according to their chromosomal abnormalities are described in Table 1.

\section{Del(13)(q14) versus PC proliferation and apoptosis}

$\mathrm{Ki}-67 \mathrm{MoAb}$ staining was performed in 78 patients. The 
percentage of Ki-67-positive PC ranged from 0 to $26 \%$ (median: $3.2 \%$ ). Annexin- $V$ staining was carried out in 69 cases and the percentage of annexin- $\mathrm{V}$-positive $\mathrm{PC}$ ranged from 0 to $55 \%$ (median: $8.1 \%$ ). The percentage of $\mathrm{Ki}-67$ expression and annexin-V labeling in PC according to clinical and laboratory characteristics is described in Table 2.

When the correlation between the percentage of PC carrying del(13)(q14) and proliferation was calculated, a weak correlation was found between $\mathrm{Ki}-67$ expression and the proportion of cells carrying del(13)(q14) $(r=0.22, \mathrm{P}=$ $0.058)$. This finding persisted even when all cases with additional $\mathrm{t}(4 ; 14)(\mathrm{p} 16.3 ; q 32)$ and $\operatorname{del}(17)(\mathrm{p} 13)$ were excluded in order to examine only the effect of del(13)(q14) on Ki-67 expression $(r=0.23, \mathrm{P}=0.06)$.

A cut-off of $8 \%$ was used for Ki-67 expression $(11,12)$. Based on the ROC curve, we determined that the best threshold for the percentage of PC with del(13)(q14) that was associated with $\mathrm{Ki}-67$ above $8 \%$ was greater than $75 \%$.
Indeed, whereas $54.5 \%$ of the cases with del(13)(q14) in at least $80 \%$ of their PC showed Ki-67 expression above $8 \%$, only $4 \%$ of patients carrying del(13)(q14) in less than $80 \%$ of their PC showed Ki-67 expression above $8 \%(\mathrm{P}$ $<0.001$ ).

No correlation was detected between del(13)(q14) and annexin- $\mathrm{V}$ staining $(r=0.05, \mathrm{P}=0.69)$.

Due to the small number of cases positive for $t(4 ; 14)$ (p16.3;q32) $(\mathrm{N}=8)$ or $\operatorname{del}(17)(p 13)(N=6)$ in our sample, no attempt was made to establish a correlation between proliferative and apoptotic indexes and the proportion of cells with these abnormalities.

\section{Survival analysis}

The overall survival from diagnosis was analyzed, and a statistically significant difference was observed between patients with $\mathrm{t}(4 ; 14)(\mathrm{p} 16.3 ; \mathrm{q} 32)$ and all other patients (estimated 3-year OS of 25 vs $62.2 \%$, respectively, $\mathrm{P}=$

Table 1. Characteristics of patients according to chromosomal abnormalities.

\begin{tabular}{|c|c|c|c|c|c|}
\hline & $\begin{array}{l}\text { All patients } \\
(\mathrm{N}=92)\end{array}$ & $\begin{array}{c}\text { Normal [normal 13q14 } \\
\text { and } 17 \mathrm{p} 13 \text {, and absence } \\
\text { of } t(4 ; 14)](\mathrm{N}=40)\end{array}$ & $\begin{array}{c}\operatorname{del}(13)(q 14) \text { [normal } \\
17 p 13 \text { and absence of } \\
t(4 ; 14)](N=32)\end{array}$ & $\begin{array}{l}t(4 ; 14) \\
(N=8)\end{array}$ & $\begin{array}{c}\operatorname{del}(17)(p 13) \\
(N=6)\end{array}$ \\
\hline \multicolumn{6}{|l|}{ Age (years) } \\
\hline Median (range) & $63.5(36-93)$ & $63.5(36-93)$ & $65(36-79)$ & $59(54-73)$ & $57.5(43-76)$ \\
\hline \multicolumn{6}{|l|}{ ISS } \\
\hline $\mathrm{I} / \mathrm{II}$ & $50 \%$ & $57.1 \%$ & $46.4 \%$ & $28.6 \%$ & $66.7 \%$ \\
\hline III & $50 \%$ & $42.9 \%$ & $53.6 \%$ & $71.4 \%$ & $33.3 \%$ \\
\hline \multicolumn{6}{|l|}{ Hemoglobin } \\
\hline Median (range), g/dL & $9.0(4.4-16.1)$ & $9.2(5.6-13.4)$ & $8.35(4.4-16.1)$ & $7.8(6.8-12.7)$ & $11.2(9.1-13.0)$ \\
\hline$<10.0 \mathrm{~g} / \mathrm{dL}$ & $64 \%$ & $60 \%$ & $68.8 \%$ & $87.5 \%$ & $33.3 \%$ \\
\hline \multicolumn{6}{|l|}{ Creatinine } \\
\hline Median (range), mg/dL & $1.2(0.38-15.6)$ & $1.2(0.56-12.2)$ & $1.3(0.38-15.6)$ & $1.6(0.81-5.2)$ & $1.2(0.7-2.4)$ \\
\hline$\geq 2.0 \mathrm{mg} / \mathrm{dL}$ & $29.1 \%$ & $25 \%$ & $34.4 \%$ & $37.5 \%$ & $16.7 \%$ \\
\hline \multicolumn{6}{|l|}{ Calcium } \\
\hline Median (range), mg/dL & $9.9(7.7-15.6)$ & $9.9(7.7-15.1)$ & $10.0(8.7-13.5)$ & $12.2(8.9-15.6)$ & $9.5(8.8-11.3)$ \\
\hline$\geq 10.5 \mathrm{mg} / \mathrm{dL}$ & $41.6 \%$ & $38.9 \%$ & $42.9 \%$ & $62.5 \%$ & $20 \%$ \\
\hline \multicolumn{6}{|l|}{ Albumin } \\
\hline Median (range), mg/dL & $3.6(1.7-5.1)$ & $3.7(2.3-4.6)$ & $3.5(1.7-4.8)$ & $3.4(2.6-3.8)$ & $3.6(3.2-5.1)$ \\
\hline$<3.5 \mathrm{~g} / \mathrm{dL}$ & $43 \%$ & $32.5 \%$ & $50 \%$ & $62.5 \%$ & $50 \%$ \\
\hline \multicolumn{6}{|l|}{$\beta_{2}$-microglobulin } \\
\hline Median (range), $\mu \mathrm{g} / \mathrm{mL}$ & $5.5(1.8-87)$ & $4.9(1.8-23)$ & $6(1.9-87)$ & $10.5(2.5-18.7)$ & $4.3(3-7.6)$ \\
\hline$\geq 3.5 \mu \mathrm{g} / \mathrm{mL}$ & $79 \%$ & $77.1 \%$ & $82.1 \%$ & $85.7 \%$ & $66.7 \%$ \\
\hline \multicolumn{6}{|l|}{ Lactate dehydrogenase } \\
\hline Median (range), U/L & $338(135-966)$ & $320(135-966)$ & $346(192-698)$ & $408(161-599)$ & $313(180-433)$ \\
\hline Above normal (>480 U/L) & $12.5 \%$ & $7.7 \%$ & $17.9 \%$ & $28.6 \%$ & $0 \%$ \\
\hline \multicolumn{6}{|l|}{ Reactive C protein } \\
\hline Median (range), mg/L & $10.9(0.36-414)$ & $12.2(1.3-260)$ & $10.6(0.88-118)$ & $36.3(1.02-101)$ & $3.9(0.57-304)$ \\
\hline Above normal (>3.0 mg/L) & $74.2 \%$ & $83.3 \%$ & $66.7 \%$ & $83.3 \%$ & $50 \%$ \\
\hline
\end{tabular}

It was not possible to study the chromosomal abnormalities in 6 of 92 patients due to hybridization failure. ISS = International Staging System (10). There were no statistical differences among groups in continuous variables $(P>0.05$, Kruskal-Wallis test). 
0.026). Del(17)(p13) was not associated with a lower estimated 3-year OS $(P=0.27)$. When cases with $t(4 ; 14)(p 16.3 ; q 32)$ and del $(17)(p 13)$ were excluded from the analysis, del(13)(q14) was also not associated with a lower estimated 3-year OS $(P=0.15$; Figure 1A).

As cases with at least $80 \%$ of cells affected by $\operatorname{del}(13)(q 14)$ showed a higher proliferative index, we compared the OS between patients carrying this abnormality in more and in less than $80 \%$ of PC. We observed that patients with more than $80 \%$ of cells affected by del(13)(q14) had a shorter median OS than patients with less than $80 \%$ of cells affected by this abnormality (estimated 3-year OS of $27.8 \mathrm{vs}$ $67.6 \%$, respectively, $\mathrm{P}=0.015)$. When cases with $t(4 ; 14)(p 16.3 ; q 32)$ and del(17)(p13) were excluded from the analysis, this difference still remained (estimated 3-year OS 32.4 vs $69.1 \%$, respectively, $\mathrm{P}=0.033$; Figure 1B). These patients were divided into three groups: group 1 [del(13)(q14) in less than $80 \%$ of PC]; group 2 [del(13)(q14) in more than $80 \%$ of $\mathrm{PC}$ and $\mathrm{Ki}-67$ expression below $8 \%$ ], and group 3 [del(13)(q14) in more than $80 \%$ of PC and Ki-67 above $8 \%$ ]. Only group 3 was associated with lower OS (estimated 3-year OS: group $1=66.2 \%$, group $2=50 \%$, and group $3=0 \%, P=0.01$ ).

The estimated 3-year EFS from diagnosis was similar in cases with $t(4 ; 14)(p 16.3 ; q 32)$, del(17) (p13), del(13)(q14), and none of the abnormalities studied $(P=0.32)$. Although del(13)(q14) in more than $80 \% \mathrm{PC}$ was also not associated with lower EFS ( $P=0.72)$, the expression of Ki-67 above $8 \%$ was associated with it $(P=0.015)$.
Table 2. Association between characteristics of patients and Ki-67 expression and annexin- $\mathrm{V}$ labeling.

\begin{tabular}{llc}
\hline & \multicolumn{1}{c}{ Ki-67 } & Annexin-V \\
\hline ISS & & \\
I/II & $2.7 \%(0-26.4 \%)$ & $8.3 \%(0.31-35.8 \%)$ \\
III & $3.2 \%(0-24.7 \%)$ & $7.3 \%(0.12-55.0 \%)$ \\
Hemoglobin & & \\
$\quad \geq 10.0 \mathrm{~g} / \mathrm{dL}$ & $2.5 \%(0.4 \%-20.9 \%)$ & $6.7 \%(0.1-35.8 \%)$ \\
$\quad<10.0 \mathrm{~g} / \mathrm{dL}$ & $3.6 \%(0-26.4 \%)$ & $9.5 \%(0.2-55.0 \%)$ \\
Creatinine & & \\
$\quad<2.0 \mathrm{mg} / \mathrm{dL}$ & $3.2 \%(0-26.4 \%)$ & $8.1 \%(0.1-55.0 \%)$ \\
$\quad 2.0 \mathrm{mg} / \mathrm{dL}$ & $3.1 \%(0-18.9 \%)$ & $8.7 \%(0.7-49.5 \%)$ \\
Calcium & & \\
$\quad<10.5 \mathrm{mg} / \mathrm{dL}$ & $3.1 \%(0-20.9 \%)$ & $9.8 \%(0.1-49.5 \%)$ \\
$\quad \geq 10.5 \mathrm{mg} / \mathrm{dL}$ & $3.6 \%(0.4-26.4 \%)$ & $6.7 \%(0.7-27.7 \%)$ \\
Albumin & & \\
$\quad \geq 3.5 \mathrm{~g} / \mathrm{dL}$ & $2.7 \%(0-26.4 \%)$ & $10.0 \%(0.1-55.0 \%)$ \\
$\quad<3.5 \mathrm{~g} / \mathrm{dL}$ & $3.7 \%(0-24.7 \%)$ & $5.8 \%(0.2-49.5 \%)$ \\
$\beta_{2}-\mathrm{microglobulin}$ & & \\
$\quad<3.5 \mathrm{\mu g} / \mathrm{mL}$ & $2.2 \%(0.8-26.4 \%)$ & $13.1 \%(0.8-35.8 \%)$ \\
$\quad \geq 3.5 \mathrm{\mu g} / \mathrm{mL}$ & $3.2 \%(0-24.7 \%)$ & $7.6 \%(0.1-55.0 \%)$ \\
Lactate dehydrogenase & & \\
$\quad \leq 480 \mathrm{U} / \mathrm{L}$ & $2.8 \%(0-24.7 \%)$ & $7.6 \%(0.1-55.0 \%)$ \\
$\quad>480 \mathrm{U} / \mathrm{L}$ & $5.0 \%(0.4-26.4 \%)^{*}$ & $21.5 \%(0.3-29.4 \%)$ \\
Reactive C protein & & $8 \%(1-32.1 \%)$ \\
$\quad 33.0 \mathrm{mg} / \mathrm{L}$ & $2.3 \%(0-6.5 \%)$ & $6.4 \%(0.1-55.0 \%)$ \\
$\quad>3.0 \mathrm{mg} / \mathrm{L}$ & $4.6 \%(0-26.4 \%)^{*}$ & \\
\hline & &
\end{tabular}

Data are reported as median with range in parentheses. ISS $=$ International Staging System (10). * $\mathrm{P}<0.05$, Ki-67 expression of above normal data compared to normal data (Kruskal-Wallis test).
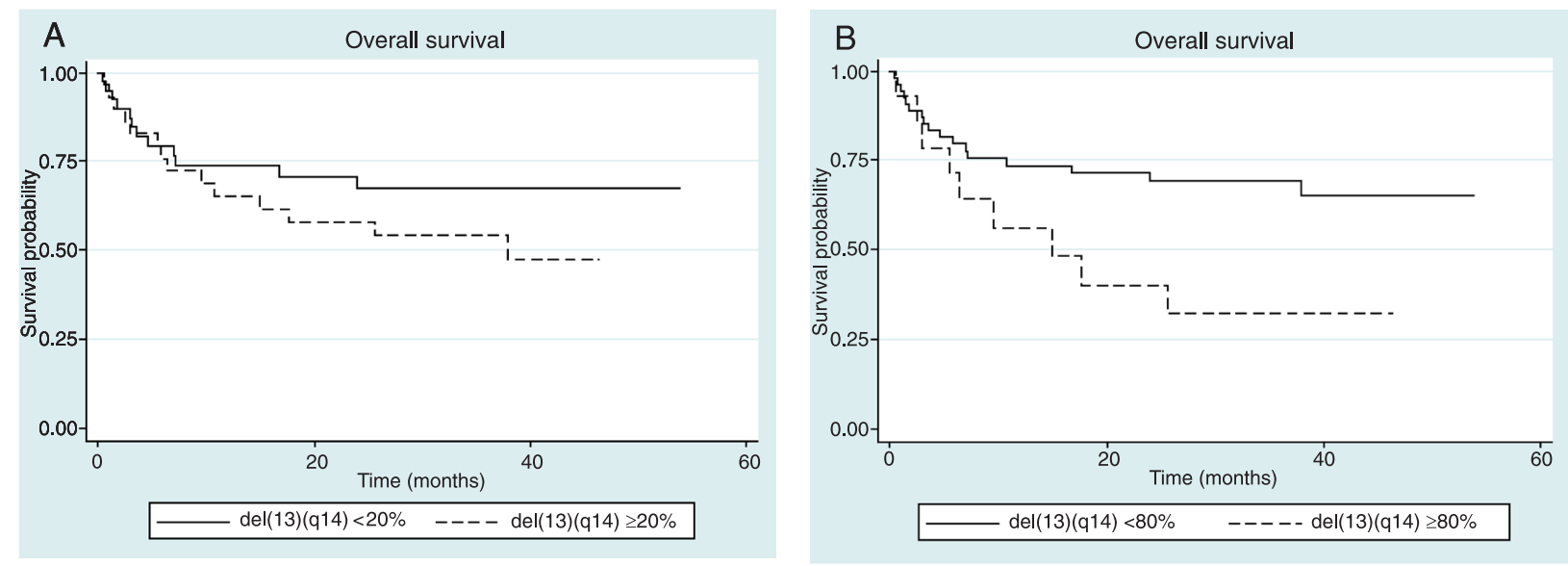

Figure 1. $A$, Overall survival $(\mathrm{OS})$ in patients without del(13)(q14) $(\mathrm{N}=40)$ and with del(13)(q14) $(\mathrm{N}=32)$ and not $\mathrm{t}(4 ; 14)(\mathrm{p} 16.3 ; \mathrm{q} 32)$ nor del(17p13) $(P=0.15$, log-rank test). $B$, OS in patients harboring del(13)(q14) in less $(N=58)$ and in more $(N=14)$ than $80 \%$ of their plasma cells and not $t(4 ; 14)(p 16.3 ; q 32)$ or del(17)(p13) $(P=0.033$, log-rank test). 


\section{Discussion}

The prevalence of del(13)(q14) and del(17)(p13) was similar to that observed in studies carried out in other countries. However, $t(4 ; 14)(p 16.3 ; q 32)$ was observed in a lower percentage of patients than in other studies (1). In the present study, $\mathrm{t}(4 ; 14)(\mathrm{p} 16.3 ; q 32)$ was associated with a very dismal prognosis, with almost half the patients dying immediately after diagnosis, before starting treatment. This suggests that, in São Paulo and perhaps in Brazil, there may be even more patients with this chromosomal abnormality, but they may die before being diagnosed due to unfavorable socioeconomic conditions, leading to under-detection of this chromosomal abnormality in our population.

We also observed that del(13)(q14) was associated with a poorer outcome when present in more than $80 \%$ of myeloma cells. Only 15.4 and $18.9 \%$ of patients with del(13) (q14) in more than and in less than $80 \%$ of PC, respectively, received ASCT (data not shown). Therefore, we could not detect any significant difference in therapy administered to the two groups that would justify this difference.

Our results suggest that, in the group of patients evaluated in this study, the proportion of cells with del(13)(q14) was more important for determining prognosis than the raw presence of this abnormality in myeloma cells. Since the late nineties, the presence of $\operatorname{del}(13)(q 14)$ in myeloma cells has been associated with a poor prognosis $(13,14)$, although Avet-Loiseau et al. (15) and Gutierrez et al. (16) showed that most of the prognostic power of this abnormality was related to its frequent association with $\mathrm{t}(4 ; 14)$ (p16.3;q32) and del(17)(p13). Despite the limited value of $\operatorname{del}(13)(q 14)$ as a sole abnormality in the determination of prognosis, we decided to evaluate its association with the proliferative and apoptotic indexes of plasma cells in order to unveil if this abnormality has any importance in the biology of the disease.

While Gastinne et al. (11) did not detect an association between del(13)(q14) and increased cell proliferation, Fonseca et al. (14) and Zojer et al. (13) reported a positive relationship between del(13)(q14) and higher growth fraction (determined by Ki-67 staining or S-phase). However, these studies did not report the status of other important cytogenetic abnormalities such as del(17)(p13), t(4;14)(p16.3;q32) or t $(14 ; 16)(q 32 ; q 23)$. A recent study showed an association of higher PC proliferative index with $\operatorname{del}(13)(q 14)$ but not

\section{References}

1. Fonseca R, Barlogie B, Bataille R, Bastard C, Bergsagel $\mathrm{PL}$, Chesi M, et al. Genetics and cytogenetics of multiple myeloma: a workshop report. Cancer Res 2004; 64: 15461558.

2. Fonseca R, Bergsagel PL, Drach J, Shaughnessy J, Gutierrez N, Stewart K, et al. International Myeloma Working Group molecular classification of multiple myeloma: spotlight with other chromosomal abnormalities (17).

Gastinne et al. (11) and Alexandrakis et al. (12) previously described a cut-off of $8 \%$ for Ki-67 expression for prognosis in MM. Our study showed that there is an association between del(13)(q14) in more than $80 \%$ of PC and a higher $\mathrm{Ki}-67$ expression (>8\%), and that this association persisted even when patients with $t(4 ; 14)(p 16.3 ; q 32)$ and $\operatorname{del}(17)(p 13)$ were excluded from the analysis. Also, the lower OS in the group of patients with more than $80 \%$ of PC affected by del(13)(q14) was observed only in cases with high Ki-67 expression. Thus, probably the cause of this unfavorable prognosis is not merely the presence of del(13) (q14) in PC, but also their higher proliferative index.

One hypothesis is that del(13)(q14) may be involved in the allelic loss of a tumor suppressor gene. As suggested by Fonseca et al. (14), additional mechanisms could lead to inactivation of the second allele, resulting in a complete loss of normal function of the affected gene, selecting these cells to become more proliferative. Consequently, the cells with del(13)(q14) would preferentially accumulate. On the other hand, when the majority of PC are affected by del(13)(q14), but the cause is not a proliferative advantage of these cells, the prognosis is not unfavorable. As far as we know, correlation between $\operatorname{del}(13)(q 14)$ and apoptosis has not been studied before and our results suggest that del(13)(q14) is not important for cell apoptosis control. Other genetic mechanisms may be more relevant for this biologic feature than $13 q 14$ status.

Another finding of the present study was that patients with del(17)(p13) did not have an unfavorable prognosis. This may be due to the low number of patients studied or to the low proportion of cells with this abnormality in the affected patients (median $=35 \%$, range $=21-76 \%$ ). These findings strengthen those described by Avet-Loiseau et al. $(15,18)$, who suggested that cases with more than $74 \%$ of plasma cells affected by $\operatorname{del}(13)(q 14)$ or with more than $60 \%$ of plasma cells affected by del(17)(p13) were characterized by a worse outcome than patients harboring these abnormalities in a smaller proportion of cells.

\section{Acknowledgments}

Research supported by the Hematology Service, Hospital das Clínicas, Faculdade de Medicina da Universidade de São Paulo. review. Leukemia 2009; 23: 2210-2221.

3. Tan D, Teoh G, Lau LC, Lim A, Lim TH, Yap KC, et al. An abnormal nonhyperdiploid karyotype is a significant adverse prognostic factor for multiple myeloma in the bortezomib era. Am J Hematol 2010; 85: 752-756.

4. Yuregir OO, Sahin FI, Yilmaz Z, Kizilkilic E, Karakus S, Ozdogu $\mathrm{H}$. Fluorescent in situ hybridization studies in multiple 
myeloma. Hematology 2009; 14: 90-94.

5. Huang SY, Yao M, Tang JL, Tsay W, Lee FY, Liu MC, et al. Clinical significance of cytogenetics and interphase fluorescence in situ hybridization analysis in newly diagnosed multiple myeloma in Taiwan. Ann Oncol 2005; 16: 1530-1538.

6. Kyle RA, Rajkumar SV. Multiple myeloma. Blood 2008; 111: 2962-2972.

7. Ahmann GJ, Jalal SM, Juneau AL, Christensen ER, Hanson CA, Dewald GW, et al. A novel three-color, clone-specific fluorescence in situ hybridization procedure for monoclonal gammopathies. Cancer Genet Cytogenet 1998; 101: 7-11.

8. European Myeloma Network. Recommendations from workshop held at the Royal Marsden Hospital LoM12. Recommendations for FISH in multiple myeloma. Available at [http:// www.cytogenetics.org.uk/prof_standards/myeloma.htm].

9. Jacob MC, Favre M, Bensa JC. Membrane cell permeabilization with saponin and multiparametric analysis by flow cytometry. Cytometry 1991; 12: 550-558.

10. Greipp PR, San Miguel J, Durie BG, Crowley JJ, Barlogie $\mathrm{B}$, Blade $\mathrm{J}$, et al. International staging system for multiple myeloma. J Clin Oncol 2005; 23: 3412-3420.

11. Gastinne T, Leleu X, Duhamel A, Moreau AS, Franck G, Andrieux J, et al. Plasma cell growth fraction using Ki-67 antigen expression identifies a subgroup of multiple myeloma patients displaying short survival within the ISS stage I. Eur J Haematol 2007; 79: 297-304.

12. Alexandrakis MG, Passam FH, Kyriakou DS, Dambaki K, Niniraki M, Stathopoulos E. Ki-67 proliferation index: correlation with prognostic parameters and outcome in multiple myeloma. Am J Clin Oncol 2004; 27: 8-13.
13. Zojer N, Konigsberg R, Ackermann J, Fritz E, Dallinger S, Kromer E, et al. Deletion of 13q14 remains an independent adverse prognostic variable in multiple myeloma despite its frequent detection by interphase fluorescence in situ hybridization. Blood 2000; 95: 1925-1930.

14. Fonseca R, Harrington D, Oken MM, Dewald GW, Bailey RJ, Van Wier SA, et al. Biological and prognostic significance of interphase fluorescence in situ hybridization detection of chromosome 13 abnormalities (delta13) in multiple myeloma: an eastern cooperative oncology group study. Cancer Res 2002; 62: 715-720.

15. Avet-Loiseau H, Attal M, Moreau P, Charbonnel C, Garban F, Hulin C, et al. Genetic abnormalities and survival in multiple myeloma: the experience of the Intergroupe Francophone du Myelome. Blood 2007; 109: 3489-3495.

16. Gutierrez NC, Castellanos MV, Martin ML, Mateos MV, Hernandez JM, Fernandez M, et al. Prognostic and biological implications of genetic abnormalities in multiple myeloma undergoing autologous stem cell transplantation: $\mathrm{t}(4 ; 14)$ is the most relevant adverse prognostic factor, whereas RB deletion as a unique abnormality is not associated with adverse prognosis. Leukemia 2007; 21: 143-150.

17. Li C, Chen L, Gao X, Qu X, Shen W, Yang R, et al. Plasma cell labeling index correlates with deletion of $13 q 14$ in multiple myeloma. Leuk Lymphoma 2011; 52: 260-264.

18. Avet-Loiseau H, Leleu X, Roussel M, Moreau P, GuerinCharbonnel C, Caillot D, et al. Bortezomib plus dexamethasone induction improves outcome of patients with $t(4 ; 14)$ myeloma but not outcome of patients with del(17p). J Clin Oncol 2010; 28: 4630-4634. 